\title{
The Earth's troposphere: absorption and refraction effects on the propagetion of radio waves
}

\author{
Javier Alcolea* \\ Observatorio Astronómico Nacional (Spain) \\ E-mail: j.alcolea@oan.es
}

In this chapter we will review the main effects that the Earth's atmosphere introduce in the radio observations. I will focus on the effects due to the neutral component of the atmosphere, which are mainly due to its lowest and densest layers, the troposphere. These layers are particularly important in the upper frequency end of the radio domain, i.e. at $\mathrm{cm}-, \mathrm{mm}-$, and sub-mm wavelengths.

2nd MCCT-SKADS Training School. Radio Astronomy: fundamentals and the new instruments August 26- September 4, 2008

Sigüenza, Spain

* Speaker. 


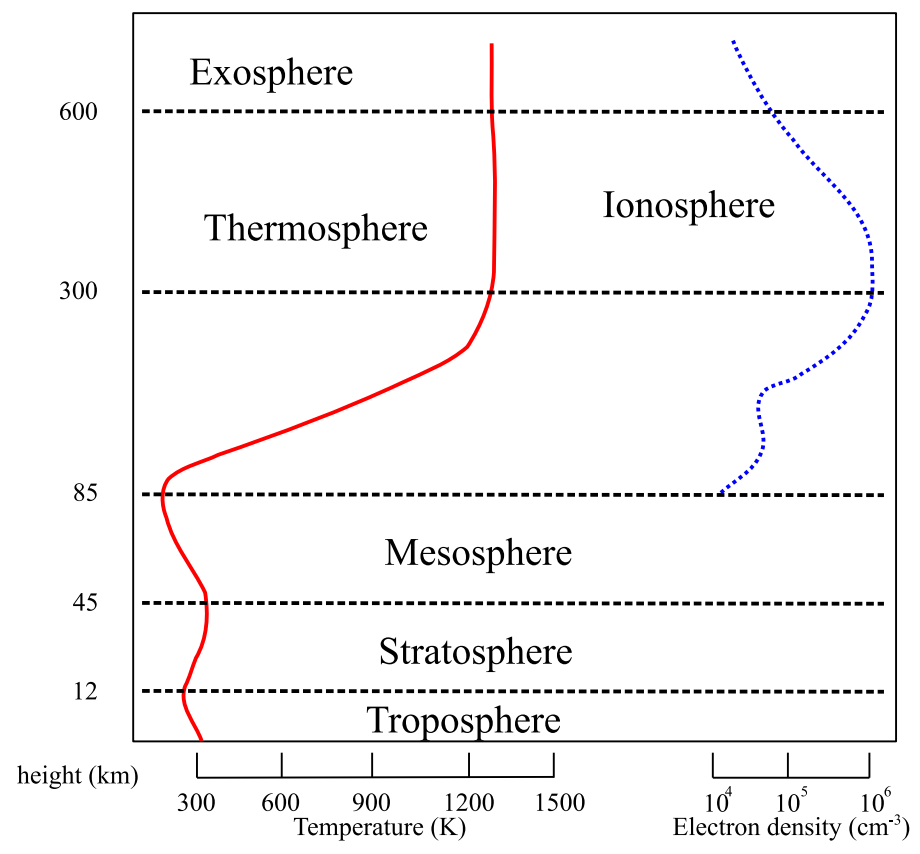

Figure 1: Sketch showing the structure and different layers of the Earth's atmosphere.

\section{Introduction: The Earth's Atmosphere}

The Atmosphere is the gas cocoon that surrounds the solid Earth. It extends up to heights of $10,000 \mathrm{~km}$ (from the Earth surface) in its outer layers. Close to the solid surface of the Earth, the atmosphere is mostly made of molecular gas, whereas in its outermost layers partially ionized gas dominates. The atmosphere is divided into several layers along its height. The separation between the different layers is made based on the dependence of the temperature with the altitude, the sign of the temperature gradient, and on its composition (degree of ionization, etc., see Fig. 1).

The existence of the atmosphere has some obvious pros: it moderates the temperature changes between day and night; it partially blocks dangerous (for the existence of life) solar radiation from penetrating down to the Earth surface; it allows the breathing of terrestrial animals and plants and the photosynthesis process; it allows the existence of the water cycle; it warms a little bit the Earth's temperature thanks to the original (not the man made) "Greenhouse effect". However, the atmosphere may also represent a major drawback for ground based observational Astronomy: it absorbs part of the incoming radiation from outer space; it disperses and refracts the radiation that penetrates; and it also introduces extra delays in the propagation of the incoming radiation.

\section{The Earth's troposphere}

At lower frequencies, cm-wavelengths and below, the main effect in EMW propagation comes from the ionized part of the atmosphere: the ionosphere. These effects have been discussed already elsewhere in these proceedings. At higher frequencies, $\mathrm{cm}, \mathrm{mm}$, submm-wavelengths, IR, visible, UV and beyond, the main effect in EMW propagation is due to the lowest and densest layer of the atmosphere: the troposphere. 
The troposphere mainly consist in molecular and noble gases, whit traces droplets, ice crystals, aerosols, dust particles and other small particles (e.g. bacteria, ashes). If we forget about these droplets and small particles we have the so called "clean air", consisting in just a mixture of gases.

The "clean air" is divided into "wet clean air" (or just wet air) and "dry clean air" (or just dry air). Wet air is just the content of water vapor in the air, while the dry air accounts for all the rest of the gas components of the troposphere. This separation is made because, by far, the most variable component of the clean air is water vapor. In this way we separate the air in a nearly constant and a highly variable components.

In average, the standard composition of the dry air in the troposphere is: $\mathrm{N}_{2} 78.1 \%$ (in volume), $\mathrm{O}_{2} 20.9 \%, \mathrm{Ar} 0.93 \%, \mathrm{CO}_{2} 0.033 \%$, $\mathrm{Ne} 0.0018 \%$, He $0.0005 \%$, and traces of other minor components. The wet air, i.e. $\mathrm{H}_{2} \mathrm{O}$ can be highly variable, though it never surpasses $1 \%$ in mass of the total content of clean air. Normally the $\mathrm{H}_{2} \mathrm{O}$ content is measured in units of the total amount of precipitable water vapor "pwv", which is given in mm, or in terms of its partial pressure, $p_{\mathrm{w}}$.

Roughly speaking, the dry massive component of the air is in hydrostatic equilibrium, hence following the equations:

$$
\frac{d p}{d h}=-g p \quad ; \quad p=\rho \frac{R T^{\mathrm{e}}}{M_{\mathrm{a}}}
$$

where $g$ is the gravitational acceleration on Earth, $p$ is the pressure, $\rho$ is the particle density, $T^{\mathrm{e}}$ is the temperature, and $M_{\mathrm{a}}$ is the average mass of the air molecules. $R$ is the "gas constant". In the standard model for the troposphere, the temperature decreases linearly with the altitude $h$

$$
T^{\mathrm{e}}=T_{0}^{\mathrm{e}}-b\left(h-h_{0}\right)
$$

If we just consider small changes in altitude we find Laplace's hydrostatic equation.

$$
\frac{\rho}{\rho_{0}}=\frac{p}{p_{0}}=\exp -h / H_{0} \quad ; \quad H_{0}=\frac{R T^{\mathrm{e}}}{M_{\mathrm{a}} g}=8.4(\mathrm{~km}) T(\mathrm{~K}) / 288
$$

$H_{0}$ is named the scale height of the atmosphere. For water vapor, i.e., the wet air, the scale height is smaller, $\sim 2.5 \mathrm{~km}$, because it is dominated by fast evaporation/condensation processes and not by the hydrostatic equilibrium equations.

\section{Transmission and absorption across the troposphere}

Since the Earth's troposphere is not empty, any incoming astronomical signal reaching the Earth will obey the equation of radiative transfer (see the contribution on EM fundamentals and the equation of radiative transfer in these proceedings). Assuming a constant temperature for the atmosphere, we obtain the equation

$$
T_{\mathrm{a}}=T_{\mathrm{s}} e^{-\tau}+T_{\mathrm{atm}}\left(1-e^{-\tau}\right)
$$

where $T_{\mathrm{a}}$ is the intensity detected by the telescope (in units of noise temperature), $T_{\mathrm{s}}$ is the equivalent temperature of the incoming radiation, $T_{\text {atm }}$ is the real temperature of the atmosphere, and $\tau$ is its corresponding opacity. 
Assuming an homogeneous atmosphere, we can express the opacity along the line of sight $\tau$ in terms of the opacity at the zenith (the position just above us) $\tau_{0}$ and the zenithal angle $z$ (the angle between the line of sight and the zenith)

$$
\tau=\tau_{0} X(z)
$$

where $X(z)$ is the so called "air mass" number (amount of air along the path in units of the amount of air in the zenithal direction). In the plane parallel approximation for the atmosphere, the air mass is given by the simple expression $X(z)=\sec (z)$. A more accurate expression for $X(z)$ y given by the formula

$$
X(z)=\frac{1}{\int_{0}^{\infty} \rho(h) d h} \int_{0}^{\infty} \frac{\rho(h)}{\sqrt{1-\left(\frac{R}{R+h} \frac{n(0)}{n(h)} \sin ^{2}(z)\right.}} d h
$$

where $R$ is the Earth radius, $h$ is the height above the Earth surface, and $n(h)$ is the index of refraction at the height $h$.

In the formula

$$
T_{\mathrm{a}}=T_{\mathrm{s}} e^{-\tau}+T_{\mathrm{atm}}\left(1-e^{-\tau}\right)
$$

the zenith opacity depends on the frequency of the incoming radiation.

$$
\tau(v)=X(z) \tau_{0} \phi(v)
$$

For a molecule to absorb photons of any particular frequency it must have a transition near that frequency. At radio wavelengths, most of the molecular transitions of major species present in the atmosphere are pure rotational lines. At higher frequencies, vibrational and electronic transitions of $\mathrm{H}_{2} \mathrm{O}, \mathrm{CO}_{2}, \mathrm{O}_{2}$ an $\mathrm{O}_{3}$ makes the sky opaque except for the well known NIR and visible windows (see Fig. 2).

Coming back to the radio domain, of all major components of clean air, just $\mathrm{H}_{2} \mathrm{O}$ and $\mathrm{O}_{3}$ (ozone), have a permanent electrical dipole moment. All the rest major components are either homo-nuclear $\left(\mathrm{N}_{2}, \mathrm{H}_{2}\right)$ of linear symmetric species $\left(\mathrm{CO}_{2}\right)$, and therefore their pure rotational transitions are forbidden.

$\mathrm{O}_{2}$ is also homo-nuclear molecule, but its ground electronic state is not a singlet but a triplet ${ }^{3} \Sigma$, resulting in a large magnetic dipole moment. These (dipolar magnetic) transitions are not so stronger as those of water and ozone, but since $\mathrm{O}_{2}$ is much more abundant, its contribution is also important. This is way the tropospheric absorption at $\mathrm{cm}_{-}, \mathrm{mm}-$, and sub-mm wavelengths is mainly due to water vapor, oxygen and ozone, in that order. Of course there are many other so called "minor components" that also contribute to the atmosphere opacity in the radio domain, but in much less importance.

There are additional minor contributions from ice crystals and droplets (of water and other species). There is also a "dry" pseudo-continuum absorption level due to absorption induced by collisions in symmetric diatomic molecules $\left(\mathrm{N}_{2}\right.$ and $\left.\mathrm{O}_{2}\right)$, and a wet continuum too.

Resonant (line) absorption is due to the molecular transitions of the atmospheric species. In the troposphere, the collisional broadening (due to the pressure of the gas) dominates and determines 


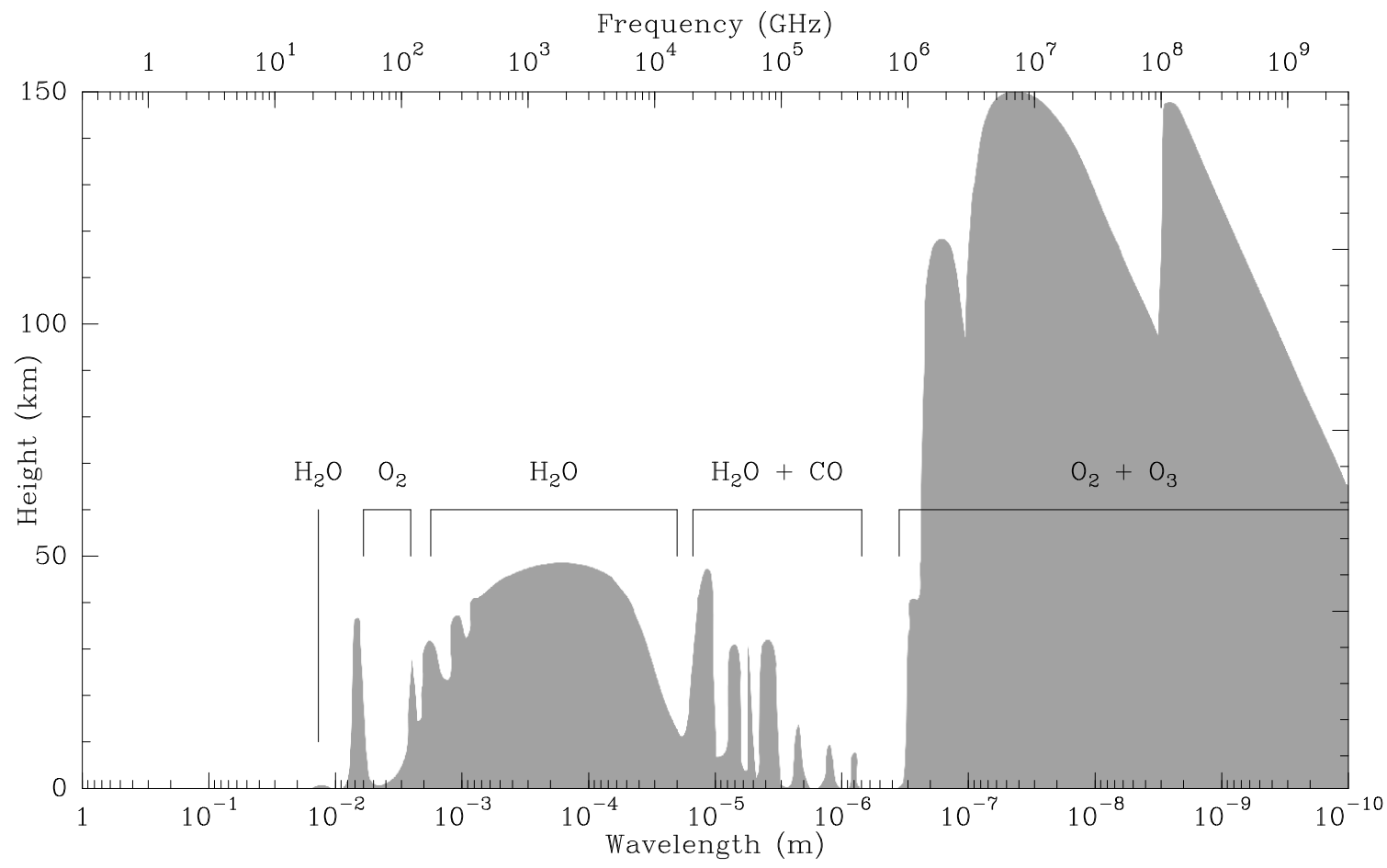

Figure 2: Height above the sea level at which the transmission of the atmosphere is $1 / 2$. We can clearly see the radio, visible and NIR windows. The main species responsible for the atmospheric absorption at each wavelength/frequency are also indicated.

the widths and shapes of these lines. If the collisions are much faster than the spontaneous radiative decays, the line-shapes (profiles) of the transitions are given by the Van Vleck and Weisskopf profile

$$
\phi\left(v, v_{0}\right)=\frac{v \Delta v}{\pi v_{0}}\left(\frac{1}{(\Delta v)^{2}+\left(v-v_{0}\right)^{2}}+\frac{1}{(\Delta v)^{2}+\left(v+v_{0}\right)^{2}}\right)
$$

where $\Delta v$ is the broadening parameter.

$$
\Delta v(p, T)=\Delta v_{0}\left(p_{0}, T_{0}\right) \frac{p}{p_{0}}\left(\frac{T_{0}}{T}\right) \gamma
$$

On the contrary, when pressure gets very low Doppler broadening due to random thermal motions becomes important, and the profiles of the lines are the convolution of a Lorentzian with a Gaussian: a Voigt profile.

\section{Calibration of radio observations}

If we observe a radio source from the ground, our radiometer (or receiver) will receive a signal given by the equation

$$
T_{\mathrm{a}}=T_{\mathrm{Rx}}+\eta_{1} T_{\mathrm{s}} e^{-\tau_{0}(v) X(z)}+\eta_{1} T_{\mathrm{atm}}\left(1-e^{-\tau_{0}(v) X(z)}\right)+T_{\mathrm{amb}}\left(1-\eta_{1}\right)
$$

where $T_{\mathrm{Rx}}$ is the receiver noise (also in units of noise temperature), $T_{\mathrm{amb}}$ is the ambient temperature, and $\eta_{1}$ is the coupling factor of the antenna to the sky (the factor $1-\eta_{1}$ thus measures the fraction 
of the signal that we pick up from the ground). However we are interested in scale units above the atmosphere, this is corrected for the attenuation of the sky, the so called $T_{\mathrm{a}}^{\star}$. Therefore, after correcting for these effects we obtain

$$
T_{\mathrm{a}}^{\star}=T_{\mathrm{a}} \frac{e^{\tau_{0}(v) X(z)}}{\eta_{1}}=T_{\mathrm{Rx}} \frac{e^{\tau_{0}(v) X(z)}}{\eta_{1}}+T_{\mathrm{s}}+T_{\mathrm{atm}}\left(e^{+\tau_{0}(v) X(z)}-1\right)+T_{\mathrm{amb}}\left(\frac{1}{\eta_{1}}-1\right) e^{\tau_{0}(v) X(z)}
$$

The sensitivity $\sigma$ of the radiometer is given by the classical formula

$$
\sigma=\frac{\sqrt{2} T_{\text {sys }}}{\sqrt{t \Delta v}}
$$

where $t$ is the integration time and $\Delta v$ is the spectral resolution. $T_{\text {sys }}$ is the power detected by our radiometer in units of noise temperature. Neglecting the contribution of the radio source, whose power is by far much less that any other term in the equation, we obtain that the system noise temperature $T_{\text {sys }}$, in equivalent units above the atmosphere, is given by the expression

$$
T_{\mathrm{sys}}=T_{\mathrm{Rx}} \frac{e^{\tau_{0}(v) X(z)}}{\eta_{1}}+T_{\mathrm{atm}}\left(e^{\tau_{0}(v) X(z)}-1\right)+T_{\mathrm{amb}}\left(\frac{1}{\eta_{1}}-1\right) e^{\tau_{0}(v) X(z)}
$$

This is why is so important to build receivers with low noise $T_{\mathrm{Rx}}$, but at the same time, locate them in telescopes with low opacity at the frequency of work.

To calibrate the observations, compensating for the atmosphere attenuation, we need to measure the sky opacity. This is done by measuring the sky emissivity off source

$$
T_{\mathrm{a}}=T_{\mathrm{Rx}}+\eta_{1} T_{\mathrm{atm}}\left(1-e^{-\tau_{0}(v) X(z)}\right)+T_{\mathrm{amb}}\left(1-\eta_{1}\right)
$$

$T_{\mathrm{Rx}}$ is obtained from measurements of hot and cold loads of known emissivity (black bodies at known temperature). $T_{\mathrm{atm}}$ and $T_{\mathrm{amb}}$ are measured from weather stations, and from measurements of the sky emissivity at several zenith angles (sky tips). $\eta_{1}$ is also measured in this way. These measurements can be done with the scientific receiver, or with special radiometers tuned to certain water lines (looking at zenith), and the aid of complex atmospheric models.

\section{Atmospheric refraction}

Another effect introduced by the lower layer of the atmosphere is the bending of the light due to the refraction effects. Since the troposphere is not empty, we can also characterize it with an index of refraction $n$. The complex part of it is the responsible of the absorption effects we have described before. The real part, in turn, describes the actual travel speed of the light across these gas layers, as well as the bending of the rays due to the refraction effects. This bending has to be taking into account for a correct pointing of the telescope in the sky.

Except for frequencies near the tropospheric absorption lines, the atmospheric refraction is independent of the frequency: i.e., the troposphere is a non dispersive media. This refraction index can be approximately expressed in the following terms

$$
(n-1)=7.7610^{-5} \frac{p}{T_{\mathrm{atm}}}+3.7310^{-1} \frac{p_{\mathrm{w}}}{T_{\mathrm{atm}}^{2}}
$$


where $p$ and $p_{\mathrm{w}}$ are respectively the total air pressure and the partial pressure of water vapor (in units of $100 \mathrm{mB}$ ) and $T_{\mathrm{atm}}$ is the temperature of atmosphere (in K). As we see, refraction largely depends on the water vapor content, that we know is also the least stable component of the troposphere.

Because of the relatively large dependence of the refraction on the water content of the air, and also due to the large humidity inhomogeneities that can develop under certain conditions, sometimes radio-telescopes suffer from what has been called "anomalous refraction". During this phenomenon, changes in the pointing of the radio-telescope, as large as $30^{\prime \prime}$, can take place in time scales of tens of seconds, which can ruin the observation (depending on the required pointing accuracy, i.e. beam size). Anomalous refraction has been observed in large telescopes, with narrow beams, in mildly poor weather conditions. It is attributed to the passage of isolated high humidity air pellets in front of the beam of the telescope.

\section{Dispersion and anomalous dispersion}

Let's take a monochromatic plane wave propagating along the $x$ direction:

$$
I(x, t)=I_{0} e^{i(k x-\omega t)} \quad ; \quad \omega=2 \pi \quad ; \quad k=\frac{2 \pi}{\lambda}
$$

This wave propagates with a phase velocity $v$, given by the equation

$$
v=\frac{\omega}{k}=\frac{c}{n}
$$

where $n$ is the refraction index. If $n$ does not depend on $k$ (or $v$ ), all monochromatic waves travel at the same speed, and non-monochromatic waves are not distorted. We say that the medium is non-dispersive.

If $n$ depends on $k$ (or $v$ ), pure monochromatic waves will not all travel at the same speed, and non-monochromatic waves will be distorted as they propagate along the medium. We say that the medium is dispersive and $\omega=\omega(k)$. Assuming that there is a linear dependence between $k$ and $\omega$ (or if not, we just take a linear approximation, that will be valid only for a range of values of $k$ ), the shape of the non-monochromatic waves will propagate at a velocity called "group velocity" $v_{\mathrm{g}}$

$$
v_{\mathrm{g}}=\frac{d \omega}{d k}=v+k \frac{d v}{d k}=v\left(1+\frac{\lambda}{n} \frac{d n}{d \lambda}\right)
$$

If $d v / d k<0$ ( or $d n / d \lambda<0$ ), i.e. the index of refraction grows with the frequency, the group velocity is smaller than phase velocity: this is called normal dispersion. If on the contrary, $d v / d k>$ 0 (or $d n / d \lambda>0$ ), i.e. the index of refraction decreases with frequency, the group velocity is larger than the phase velocity: this is called anomalous dispersion because the shape (and energy transported by the wave) propagates faster than the speed of the light in the media (but not faster than $c$ ).

For frequencies near those of the radio lines of $\mathrm{O}_{2}, \mathrm{H}_{2} \mathrm{O}$ and $\mathrm{O}_{3}$, the non-dispersive properties of the atmosphere are lost, since the travel speed does depend on the frequency. This is simply because opacity strongly depends on the frequency near the resonant absorption lines, and opacity (the imaginary part of the index of refraction) and the refraction index (the real part) must vary in a 
similar way due to the Kramers-Kroning relations. Therefore we should have normal dispersion in one side of the line, and anomalous dispersion at the other side of the line. We have a phase delay at frequencies below that of the resonant line and, a phase advance at higher frequencies.

\section{Tropospheric phase delays}

For interferometers, in addition to the absorption and refraction effects, we should also consider the delay (or phase) of the signal introduced by the atmosphere. As for the refraction, for the case of clean air, the most important source of atmospheric delay is the water content. Once more, this is very difficult to model and predict because if varies in time scales that depend on the weather conditions: humidity degree, wind, etc.

It has been empirically demonstrated that the delay due to water vapor is just proportional to the water vapor content of the atmosphere. In addition we also have an extra delay near strong absorption lines, similarly to what we have just discussed for the refraction. This is normal since these delays are just consequences of the different travel speeds depending on the value of the refraction index.

There are several ways of correcting or mitigating the effects of the atmospheric phase delay in interferometers. We can use atmospheric models in combination with weather stations. This method works up to some extent, but can not cope with the delays due to inhomogeneities in the wet air.

We can also intersperse observations of astronomical calibrators: i.e. point-like sources of well known position, and therefore for which the "due observed phase" is well known a priory. This is the normal approach, but requires that the change in phase delay between the direction of the calibrator and of the source, remains below $\approx \pi$ radians between two consecutive observations of the science target or the calibrator. Otherwise is difficult to follow the trend of the phases and be sure that there are no additional $2 \pi$ radian jumps between the different observations. This limits the angular separation between calibrator and source, the duration of the duty cycle, and does not correct for the short time de-correlation of the signal.

We can combine the data taken simultaneously at two frequencies. Since the phase delay is due to the refraction index of the sky, if we are outside an absorbing line, the medium is not dispersive and the following relation must hold:

$$
\frac{\Delta \phi\left(v_{1}\right)}{v_{1}}=\frac{\Delta \phi\left(v_{2}\right)}{v_{2}}
$$

i.e. the phase delay is proportional to the observing frequency. We can use phases derived for a lower frequency observation to correct data at higher frequencies, where they are more difficult to estimate for many reasons (larger phase delays, noisier receivers, weaker calibrators, larger atmospheric opacities). This has bee used with success in both in VLBI and connected interferometers at $\mathrm{cm}$ - and mm-wavelengths.

A new experimental method is being tried at several observatories, for instance the IRAM Plateau e Bure Interferometer. It consist in including in each antenna, a dedicated receiver measuring the water vapor content the atmosphere along the line of sight. These receivers, normally called water vapor radiometers, measure the emission of the atmosphere in some water line, to 
derive the amount of precipitable water along the lines of sight in real time. The hope is that in this way the atmospheric phase noise can be corrected in real time, diminishing the effects of the short term de-correlation of the signal, allowing the use of more distant calibrators, and the use of longer baselines at even higher frequencies.

\section{References}

[1] M. Guélin, Atmospheric Absorption in the proceedings of the $1^{\text {st }}$ IRAM Millimeter Interferometry School, Grenoble 1998, http: / /www.iram.fr/IRAMFR/IS/school .htm

[2] J.R. Pardo, Atmospheric Absorption in the proceedings of the $2^{\text {st }}$ IRAM Millimeter Interferometry School, Grenoble 2000, http: / / www. iram. fr/IRAMFR/IS/ school . htm

[3] K. Rolfs, T.L. Wilson, Tools of Radio Astronomy ( $2^{\text {nd }}$ edition), Astronomy \& Astrophysics Library, Springer Verlag. 